\title{
On the Locus of Curves with Automorphisms
}

\section{Maurizio Cornalba}

Published online: 7 June 2007

C) Springer-Verlag 2007

\section{Erratum to: Annali di Matematica (1987) 149(4):135-151 DOI 10.1007/BF01773930}

As pointed out to me by Giancarlo A. Urzúa, to whom I am very grateful, the statement of Theorem 1 on page 141 of the paper in question contains an error. To correct it, replace point iii) on lines $15-16$ with

(iii) $g^{\prime}=0, n=4, a_{4}=p-1$ (and hence $a_{2}+a_{3}=p$ ); $\sigma$ acts on $\left\{q_{1}, q_{2}, q_{3}, q_{4}\right\}$ as the product of two disjoint transpositions.

The mistake in the original proof occurs on line 30 of page 142, where it is said that "The other cases are similar". Similar indeed they are, but the conclusions are not. Here is a correct argument. Suppose $\sigma$ interchanges $q_{i}$ and $q_{j}$. Then Lemma 1 says that there is an integer $b$, with $1 \leq b<p$, such that $a_{j} \equiv b a_{i}$ and $a_{i} \equiv b a_{j}$ modulo $p$. Thus $b^{2} \equiv 1$ modulo $p$, and hence $b$ equals 1 or $p-1$. As $1=a_{1} \leq a_{2} \leq a_{3} \leq a_{4}<p$, and the $a_{i}$ add up to a multiple of $p$, if $\sigma$ interchanges $q_{1}$ with $q_{2}$ the only possibility is that $a_{1}=a_{2}=1$ and $a_{3}=a_{4}=p-1$, as correctly stated in the original proof. The same happens if $\sigma$ interchanges $q_{1}$ with $q_{3}$. If instead $\sigma$ interchanges $q_{1}$ with $q_{4}$, all one can conclude is that $a_{4}=p-1$ and $a_{2}+a_{3}=p$; the second equality is anyway implied by the first and by the fact that the sum of the $a_{i}$ is divisible by $p$.

There remains to show that the latter case does indeed occur. This is proved exactly as on lines 27-31 of page 141 of the paper. Namely, suppose that $1=a_{1} \leq a_{2} \leq a_{3} \leq a_{4}=p-1$ and that $a_{2}+a_{3}=p$. Normalize things so that $q_{1}=0, q_{2}=1, q_{3}=\zeta$, and $q_{4}=\infty$, where $\zeta$ is a complex number different from 0 and 1 . If we let $\sigma$ denote the linear fractional transformation $z \mapsto \zeta / z$, then $\sigma$ acts on $\left\{q_{1}, \ldots, q_{4}\right\}$ as required. Morerover, if we set

The online version of the original article can be found under doi:10.1007/BF01773930 .

M. Cornalba $(\varangle)$

Indirizzo dell'A.: Dipartimento di Matematica,

Università di Pavia,

Via Ferrata 1, 27100 Pavia, Italy

e-mail: maurizio.cornalba@unipv.it 
$b=p-1$, then $b a_{1} \equiv a_{4}, b a_{4} \equiv a_{1}, b a_{2} \equiv a_{3}, b a_{3} \equiv a_{2}$ modulo $p$; hence $\sigma$ lifts to an automorphism of $C$ by Lemma 1.

The error in Theorem 1 causes an error to occur also in Corollary 1 of the paper. To correct this, one merely has to replace line 4 on page 147 with

(iii) $g^{\prime}=0, n=4, a_{4}=p-1$.

The proof is unchanged.

The tables appended to the paper, which were calculated using Corollary 1, are also partially incorrect. To correct the first table all one has to do is delete all subvarieties $S\left(p, 0 ; 1, a_{2}, a_{3}, p-1\right)$. To correct the second table one needs to modify the number of 1-dimensional components of the singular locus of $M_{g}$ to $g(g+2) / 24$ if $g+1$ is a prime, and correct the total number of components accordingly. 